\title{
Biohybride Architekturen für eine effiziente Umwandlung von Licht in elektrische Energie durch Integration von Photosystem I in skalierbare makroporöse 3D-Elektroden
}

\author{
Kai Stieger, Sven Christian Feifel, Heiko Lokstein, Mahdi Hejazi, Athina Zouni, Fred Lisdat*
}

\section{Zusammenfassung}

Die Kombination von fortschrittlichen Materialien und kontrolliertem Oberflächendesign mit komplexen Proteinen aus der natürlichen Photosynthese ist derzeit eines der Hauptthemen bei der Entwicklung von Biohybridsystemen und Biophotovoltaik. In dieser Studie werden transparente makroporöse Indium-Zinn-Oxid-( $\mu$ ITO-) Elektroden mit dem trimeren Superkomplex Photosystem I (PSI) aus dem Cyanobakterium Thermosynechococcus elongatus sowie dem kleinen Redoxprotein Cytochrom $c$ (Cyt c) kombiniert, um neuartige und effiziente biohybride Photokathoden herzustellen. Mit diesen bis zu $40 \mu \mathrm{m}$ hohen 3D-Strukturen können beide Proteine in einer annähernden Monolage abgeschieden werden und die elektrische Kommunikation mit der Elektrode kann erzielt werden. Der generierte Photostrom folgt dabei linear der kontrollierbaren Schichtdicke der $\mu \mathrm{ITO}$-Elektrode, wobei Stromdichten von bis zu $150 \mu \mathrm{A} \mathrm{cm}^{-2}$ erhalten werden. Eine effiziente elektrische Kopplung der Proteine kann durch die hohe interne Quanteneffizienz von 30 \% gezeigt werden.

\section{Abstract}

The combination of advanced materials and a controlled surface design with complex proteins from natural photosynthesis is currently one of the major topics in the development of biohybrid systems and biophotovoltaic devices. In this study, transparent macroporous indium tin oxide ( $\mu \mathrm{ITO}$ ) electrodes are combined with the trimeric supercomplex, Photosystem I (PSI) from the cyanobacterium Thermosynechococcus elongatus, and the small redox protein cytochrome $c$ (cyt $c$ ) to create novel and efficient biohybrid photocathodes. With these 3D structures up to $40 \mu \mathrm{m}$ in size, both proteins can be deposited in a monolayer fashion and electrical communication with the electrode can be established. The generated photocurrent linearly follows the controllable layer thickness of the $\mu$ ITO electrode up to $40 \mu \mathrm{m}$, whereby current densities of up to $150 \mu \mathrm{A} \mathrm{cm}{ }^{-2}$ are obtained. An efficient electrical coupling of the proteins can be demonstrated by the high internal quantum efficiency of $30 \%$.

\section{Einleitung}

Der Aufbau und die Entwicklung von Photobiohybrid-Elektroden aus Schlüsselkomponenten der natürlichen Photosynthese - vor allem aus den Proteinkomplexen der Lichtenergieumwandlung - sind in den letzten Jahren verstärkt vorangetrieben worden (Czechowski et al. 2014, Sassolas et al. 2012). Diese BiohybridElektroden eignen sich für mögliche zukünftige Anwendungen wie photoschaltbare Biosensoren oder für photokatalytische oder photovoltaische Systeme und bilden die Grundlage einer biobasierten erneuerbaren Wirtschaft (Badura et al. 2011a; Plumeré
2012; Leech et al. 2012; DominguezBenetton et al. 2013; Kothe et al. 2013; Gordiichuk et al. 2014; Operamolla et al. 2015).

Von der Natur lernend, sind gerade die Licht in Ladungsträger umwandelnden Proteine der oxygenen Photosynthese von Pflanzen und Cyanobakterien von großem Interesse für die Konstruktion von neuartigen funktionellen Systemen (Wang et al. 2013). Einer der vielversprechendsten lichtumwandelnden Proteinkomplexe ist das Photosystem I (PSI), aufgrund seiner hohen Quanteneffizienz ( 100\%), einer schnellen und stabilen Ladungstrennung und einer effektiven spektralen Überlappung mit dem Sonnenlicht
(Nelson \& Yocum 2006; Wang et al. 2013). Im thermophilen Cyanobakterium Thermosynechococcus elongatus (T. elongatus) liegt PSI als trimerer Pigment-Protein-Superkomplex vor, der pro monomerer Untereinheit aus 12 unterschiedlichen Proteinuntereinheiten besteht sowie 96 Chlorophyll $a(\mathrm{Chl} a)$ und 22 Carotinoide beherbergt. Die meisten $\mathrm{Chl}$ dienen als lichtsammelnde Antennenpigmente, während $6 \mathrm{Chl}$ die Elektronentransportkette formieren (Jordan et al. 2001). Im PSI erfolgt die Ladungstrennung am luminalen Pigmentdimer, Chl a / Chl $a^{\prime}\left(\mathrm{P}_{700}\right)$, was schließlich zu einer Reduktion des stromal angeordneten terminalen Eisen-Schwefel-Clusters $\left(F_{B}\right)$ führt (Díaz- 
Quintana et al. 1998; Grotjohann \& Fromme 2005). Die lichtinduzierten Elektronen-Loch-Paare werden im natürlichen System über Cytochrom $c_{\sigma}$ (Cyt $c_{6}$ ) aufgefüllt, das $P_{700}$ reduziert - die Oxidation von PSI erfolgt durch Ferredoxin ( $\mathrm{Fdx}$ ) am $\mathrm{F}_{\mathrm{B}}$-Cluster (Cramer et al. 2006).

Die effektive elektrische Kontaktierung von PSI mit verschiedenen Elektrodenstrukturen und Materialien ist von fundamentaler Bedeutung für die Generierung einer funktionellen photobiohybriden Elektrode mit hoher Photostromausbeute (Nguyen \& Bruce 2014). Eine Vielzahl von Strategien wurde bereits veröffentlicht, die unterschiedliche Oberflächendesigns und eine unterschiedliche Oberflächenchemie verwenden (Ko et al. 2004; Kincaid et al. 2006; Ciobanu et al. 2007; Carmeli et al. 2007; Grimme et al. 2008, 2009; Yan et al. 2012; Manocchi et al. 2013). Eine deutliche Verbesserung der Elektrodenleistung erfolgt, wenn elektrische Signalketten mit Elektronen-Shuttle-Systemen oder leitfähigen Molekülen verwendet wurden (Terasaki et al. 2009; Yehezkeli et al. 2010; Efrati et al. 2013; Stieger et al. 2014; Feifel et al. 2015).
Um die Einschränkungen von 2DAnordnungen zu überwinden und um höhere Photoströme je geometrischer Elektrodenfläche zu erzeugen, wurden verschiedene Strategien entwickelt, die vorwiegend in drei Gruppen aufgeteilt werden können: Multischicht-Architekturen (Ciesielski et al. 2010; LeBlanc et al. 2012, 2014; Yehezkeli et al. 2013; Ocakoglu et al. 2014; Stieger et al. 2016), 3D-Polymergele (Badura et al. 2011a; Hartmann et al. 2014; Kothe et al. 2014; Baker et al. 2014; Gizzie et al. 2015; Zhao et al. 2015) und 3D-Elektrodenoberflächen (Terasaki et al. 2006; Ciesielski et al. 2008; Yu et al. 2015). Besonders in der letztgenannten Gruppe kann ein großes Anwendungspotential erkannt werden, da sich Einschränkungen des Elektronentransports in PSI-Multischicht-Architekturen aufgrund langer Elektronentransferwege umgehen lassen. Lichtinduzierte Ladungsträger können direkt in ein hochleitfähiges Material injiziert werden, ohne auf eine planare Fläche beschränkt zu sein. Photoaktive PSI-haltige 3D-Elektroden wurden daher entwickelt - oftmals mit Halbleitermaterialien, wie z. B. ${\mathrm{ZnO} / \mathrm{TiO}_{2}-}^{-}$

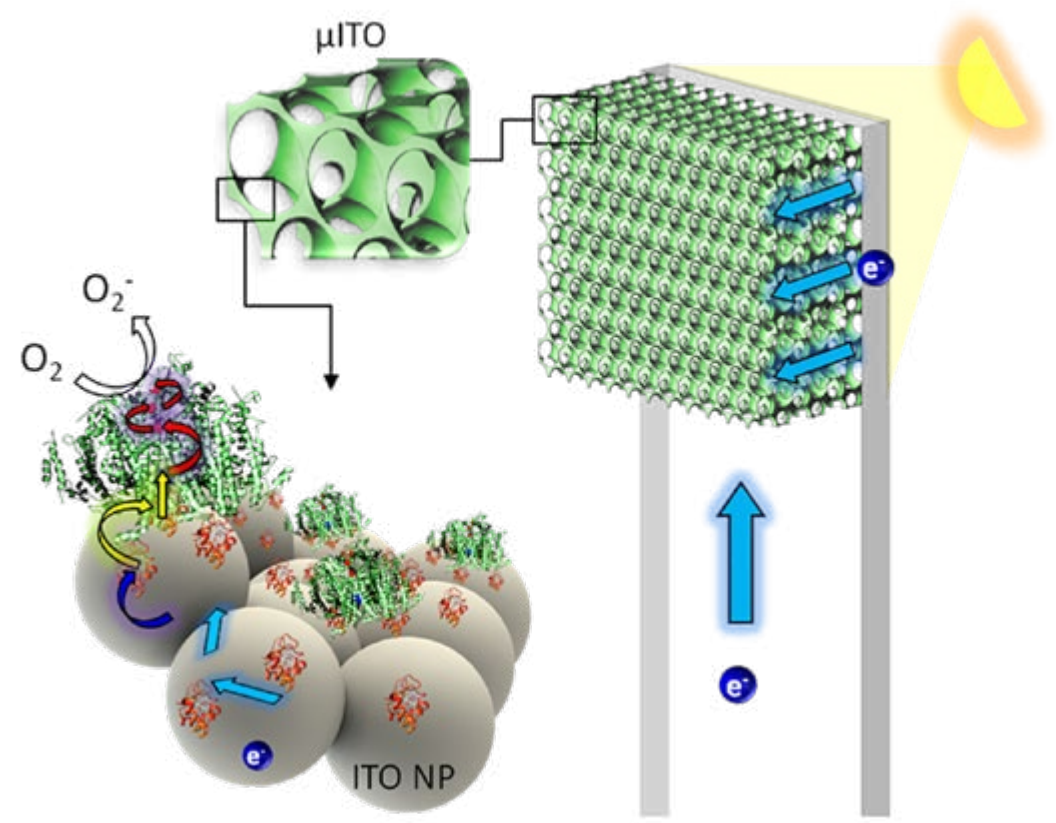

Abb. 1) Schematische Darstellung einer $\mu$ ITO-Elektrode mit integriertem Photosystem I (PSI) und Cytochrom c (Cyt c). Die uITO-Struktur zeigt eine invers opale makroporöse Struktur mit nanopartikulärer Oberflächenrauigkeit. Cyt c bindet an die Oberfläche und ermöglicht die elektrische Kontaktierung von PSI zur $\mu$ ITO-Elektrodenoberfläche. Nach der Lichtanregung fließen Elektronen aus der Basis-ITO-Elektrode in die uITO-Struktur (bestehend aus gesinterten ITO-Nanopartikeln [ITO NP]) weiter zu Cyt c, das anschließend PSI reduziert. Das photoangeregte Elektron im PSI kann auf molekularen Sauerstoff übertragen werden. Hellblaue Pfeile zeigen den intrinsischen Elektronentransfer in ITO, der dunkelblaue Pfeil zeigt den heterogenen Elektronentransfer, gelbe Pfeile zeigen den Interprotein-Elektronentransfer und rote Pfeile die intramolekulare Elektronentransferkaskade von PSI.

Nanodrähten (Mershin et al. 2012) oder nanostrukturierten $\mathrm{TiO}_{2}$-Säulen (Shah et al. 2015). Darüber hinaus konnten bereits andere lichtsammelnde Komponenten wie das Bakterienreaktionszentrum - Lichtsammelkomplex 1 (RC-LH1) mit makroporösen Silberelektroden kontaktiert werden (Friebe et al. 2016).

In dieser Studie nutzen wir die Eigenschaften einer makroporösen IndiumZinn-Oxid-Elektrode ( $\mu$ ITO). Transparente leitende Oxide (TCO) bieten den Vorteil einer hohen Transparenz bei ausreichender Leitfähigkeit. Eine direkte Elektrochemie großer Proteine an TCOs ist oftmals schwer zu realisieren. Die Ursachen dafür können vielfältig sein, wie z. B. unpassende chemische Oberflächengruppen, eine niedrige intrinsische Ladungsträgerdichte oder geringe Elektronentunnelraten. Durch eine geeignete Oberflächenmodifikation wurden jedoch effiziente funktionelle Systeme mit Enzymen nachgewiesen (Frasca et al. 2013; Sarauli et al. 2014a, b). Zusätzlich konnte Photosystem II (PSII) funktional auf solchen Oberflächen immobilisiert werden (Kato et al. 2012; Mersch et al. 2015).

In dieser Studie verwenden wir Cytochrom c (Cyt c) als molekulares Netzwerk für die elektrische Kopplung von PSI-Molekülen mit der Elektrode. Bereits zuvor konnten wir dieses Kontaktierungssystem in Form einer Mono- und Multischicht-Architektur vorstellen (Stieger et al. 2014, 2016). Cyt $c$ adsorbiert auf planarem (EI Kasmi et al. 2002; Araci et al. 2008), meso- (Frasca et al. 2010; Liu et al. 2014) und nanostrukturiertem (Schaming et al. 2012) ITO und zeigt eine ausreichend hohe heterogene Elektronentransfergeschwindigkeitskonstante $\left(k_{s}\right)$ auf diesen Materialien. Als logische Konsequenz kombinieren wir das Cyt-c-PSI-System mit einer makroporösen Indium-Zinnoxid-Elektrode ( $\mu \mathrm{ITO})$, um eine Photobiokathode mit höherer Leistung zu konstruieren. Wir charakterisieren die (photo-) elektrochemischen Eigenschaften der Elektrode hinsichtlich ihrer Effizienz und Skalierbarkeit sowie die Möglichkeiten zur Leistungsverbesserung der Licht-zu-Strom-Umwandlung. 
Ergebnisse und Diskussion

In dieser Studie nutzen wir die Transparenz und Leitfähigkeit von ITO und kombinieren sie mit PSI und Cyt c, um ein funktionelles Photokathodensystem zu entwickeln. In Abb. 1 werden der Elektrodenentwurf und die Elektronentransferwege gezeigt. Zur Herstellung einer dreidimensionalen $\mu$ ITO-Elektrode wird eine Mischung aus ITO-Nanopartikeln $\left(\right.$ ITO $\left._{\mathrm{NP}}\right)$ und Latexbeads (LB) auf ein ITO-Glas mittels Spin-Coating-Verfahren aufgebracht. Nachdem die Schichten auf der Oberfläche abgeschieden worden sind, erfolgt ein Sintervorgang bei hohen Temperaturen. Durch thermische Zersetzung der LB bildet sich eine invers opale makroporöse Struktur. Die erzeugte Hohlstruktur wird dann zunächst mit PSI inkubiert, gefolgt von einer Inkubation mit Cyt $c$, woraus sich dann eine grünlich gefärbte Elektrode ergibt. Nach der Belichtung wird ein kathodischer Photostrom erhalten, der bestätigt, dass Elektronen von der planaren ITO-Elektrode zu der abgeschiedenen porösen ITOStruktur transportiert werden, wodurch das gebundene Cyt $c$ und ferner das PSI reduziert werden. Auf der stromalen Seite des PSI können Elektronen schließlich auf einen Akzeptor in Lösung übertragen werden (hier Sauerstoff, siehe Abb. 1). Die Signalkette wird durch die Lichtanregung von PSI ein- und ausgeschaltet.

Zunächst werden die $\mu$ ITO-Elektroden mit Rasterelektronenmikroskopie (REM) und zyklischer Voltammetrie (CV) charakterisiert. REM-Messungen zeigen eine regelmäßige Struktur mit kreisförmigen Löchern, die Verbindungen zu der nächsten Schicht von entfernten Latexbeads umfassen (Abb. 2). Es werden homogene Löcher mit einem Durchmesser von $640 \pm$ $10 \mathrm{~nm}(\mathrm{n}=20)$ erhalten. Die Verbindungen zwischen den Schichten sind unterschiedlich groß und reichen von 200 bis $400 \mathrm{~nm}$. Dies gewährleistet den Zugang zu tieferen Schichten des $\mu$ ITO auch für große Proteine wie PSI. Die $\mu$ ITO-Elektroden können mit einer unterschiedlichen Anzahl von Spin-Coating-Schritten hergestellt werden, was eine definierte Einstellung der Dicke der Elektrodenstruktur ermöglicht. Die mittlere Schichtdicke
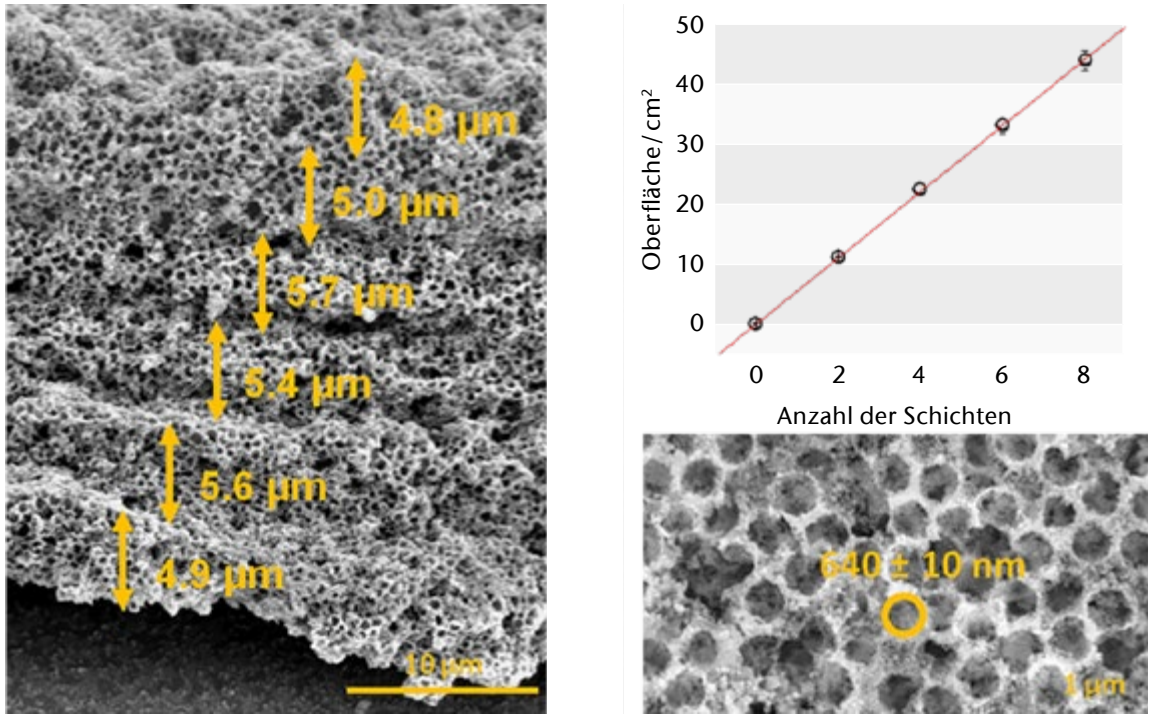

Abb. 2) Rasterelektronenmikroskopische Aufnahmen von gesinterten $\mu$ ITO-Elektroden, hergestellt durch $6 x$-Spin-

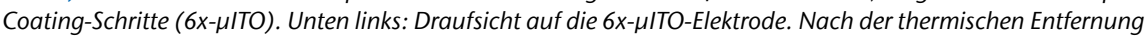
von Latexbeads kann der Lochdurchmesser auf $640 \pm 10 \mathrm{~nm}(n=20)$ bestimmt werden. Rechts: Seitenansicht der $6 x$ - $\mu$ ITO-Elektrode. Eine definierte Trennung zwischen den sukzessiv hinzugefügten Spin-Coating-Schichten mit einer Höhe von 5,2 $\pm 0,4 \mu \mathrm{m}$ / Schicht ist sichtbar. Oben links: Die elektroaktive Oberfläche nimmt in Abhängigkeit von der Anzahl der aufgetragenen Schichten zu. Die Oberfläche erhöht sich um 5,5 0,01 cm² / Schicht $\left(R^{2}=0,999, n=4\right)$

für einen Spin-Coating-Schritt beträgt 5,2 $\pm 0,4 \mu \mathrm{m}$. Die Erhöhung der elektroaktiven Oberfläche kann durch CV-Messungen überprüft werden. Da die Doppelschichtkapazität mit der Elektrodenfläche im Elektrolytkontakt skaliert, wird der elektroaktive Bereich aus dem kapazitiven Strom des planaren ITO und den verschiedenen $\mu$ ITOs berechnet. Die Größe der Oberfläche skaliert linear mit der Anzahl der Schichten $\left(R^{2}=0,999\right)$ mit einer Steigung von $5,5 \pm 0,01 \mathrm{~cm}^{2} /$ Schicht. Die Ergebnisse aus den CV- und SEM-Messungen zeigen, dass die Oberfläche und die Höhe der $\mu$ ITO-Elektroden direkt über die Anzahl der abgeschiedenen Schichten ohne Limitierungen gesteuert werden können. Darüber hinaus sind strukturelle Eigenschaften wie Lochdurchmesser, Schichtverbindungen und Gleichmäßigkeit in allen erzeugten Elektroden hoch konserviert.

Photoelektrochemische Eigenschaften von $\mu$ ITO-PSI-Cyt-c-Elektroden

Im Rahmen der Arbeit wurden verschiedene Strategien zur Kombination von PSI und Cyt $c$ mit der 3D-Elektrode untersucht. Das Verhalten dieser Elektroden unter Beleuchtung wurde als Erfolgskriterium verwendet. Neben den Ansätzen der Inkubation der Elektroden in Lösung und des Tropfengusses auf der Struktur wurde die
Reihenfolge der Proteininkubationen und der Proteinkonzentration variiert. Die höchste Photostromdichte kann erreicht werden, wenn das folgende Herstellungsverfahren angewendet wird: Zuerst wird eine konzentrierte PSI-Lösung (> $20 \mu \mathrm{M}$ ) auf die Elektrode aufgetropft und für einige Minuten inkubiert, dann wird die Elektrode mit Phosphatpuffer gewaschen. Im nächsten Schritt wird eine 1-mM-Cyt-c-Lösung aufgetropft und für einige $\mathrm{Mi}$ nuten inkubiert, gefolgt von Waschen mit Phosphatpuffer. Nach den Inkubationsschritten zeigt die grünliche Farbe der Elektrode bereits die Bindung von PSI an die $\mu$ ITO-Oberfläche an. Der zweite Inkubationsschritt mit Cyt $c$ sorgt für die Bindung des Redoxproteins an PSI und die $\mu$ ITO-Oberfläche, was zu einer Verbindung von PSI mit der Elektrode über Cyt $c$ führt. Um den Elektronentransfermechanismus einer solchen $\mu$ ITO-PSI-Cyt-c-Elektrode aufzuklären, wurden CV-Experimente durchgeführt. Sie zeigen die direkte Elektrochemie von Cyt $c$ an der $\mu$ ITOElektrode (Daten nicht gezeigt), die vergleichbar mit Ergebnissen an anderen porösen ITO-Elektroden erhalten wurden (Frasca et al. 2010). Klare Oxidations/Reduktionspeaks sind mit einer Peakseparation von $80 \mathrm{mV}$ und einer vollen Peakbreite bei einer halbmaximalen Peakbreite von $93 \mathrm{mV}$ sichtbar, was als quasi-reversibler heterogener 


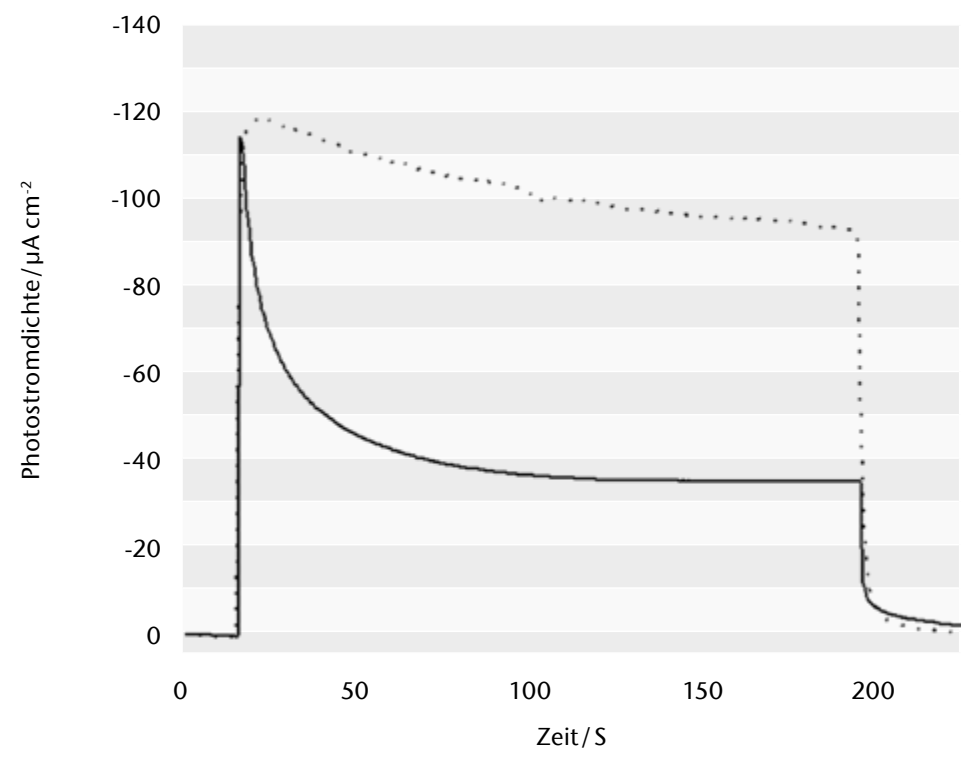

Abb. 3) Photostromdichte einer 6x- $\mu$ ITO-PSI-Cyt-c-Elektrode. Die Experimente wurden bei Raumtemperatur in Phosphatpuffer ( $5 \mathrm{mM}, \mathrm{pH}$ 7) unter Verwendung von weißem Licht $\left(20 \mathrm{~mW} \mathrm{~cm}^{-2}, 3 \mathrm{~min}\right.$ ) bei einem Potential von -100 mV vs. Ag|AgCl unter konstantem Rühren (gepunktet) und ohne (durchgängig) durchgeführt. Geometrische Elektrodenfläche: $0,2 \mathrm{~cm}^{2}$.

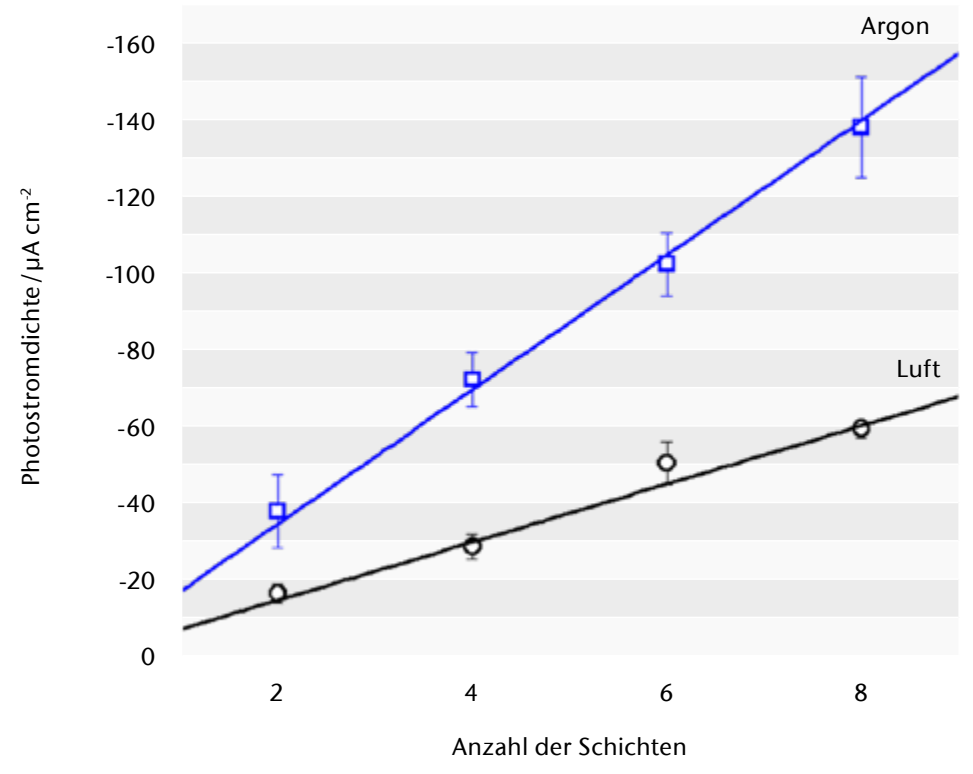

Abb. 4) Photostromdichte in Abhängigkeit von der Anzahl der aufgebrachten $\mu$ ITO-Schichten einer $\mu$ ITO-PSICyt-c-Elektrode und unterschiedlichen Sinterverfahren (PAir = Sintern unter Luft $=$ Kreise, PArgon $=$ Sintern unter Argon = Quadrate). Eine lineare Beziehung zwischen dem Photostrom und der Anzahl der Schichten wird gefunden (PArgon: 17,5 $\pm 0,4 \mu \mathrm{A} \mathrm{cm} \mathrm{cm}^{-2}$ / Schicht, $R^{2}=0,992$, PAir: 7,7 $\pm 0,2 \mu \mathrm{A} \mathrm{cm^{-2 }} /$ Schicht, $R^{2}=0,989$ ). Experimente wurden bei Raumtemperatur in Phosphatpuffer $(5 \mathrm{mM}, \mathrm{pH}$ 7) unter Verwendung von weißem Licht $(20 \mathrm{~mW} \mathrm{cm-2)}$ bei einem Potential von $-100 \mathrm{mV} \mathrm{vs.} \mathrm{Ag} \mid \mathrm{AgCl}(n=4)$ durchgeführt. Geometrische Elektrodenfläche: $0,2 \mathrm{~cm}^{2}$.

Elektronentransfer des Redoxproteins mit der Elektrode eingeordnet werden kann (Nicholson \& Shain 1964). Hierbei ist zu beachten, dass die Elektrochemie von Cyt $c$ nicht durch die Anwesenheit von PSI in der 3DElektrodenstruktur gestört wird. Unter Beleuchtung wird ein deutlicher, nur kathodischer katalytischer Strom, beginnend bei etwa $70 \mathrm{mV}$, gegen $\mathrm{Ag} \mid \mathrm{AgCl}$ nachgewiesen (Daten nicht gezeigt). Dies deutet darauf hin, dass Cyt $c$ durch die Elektrode reduziert wird, um Elektronen weiter auf PSI zu übertragen, wie bereits auf modifiziertem Gold nachgewiesen werden konnte (Stieger et al. 2014, 2016). Für ein besseres Verständnis der Limi- tierungen innerhalb einer derart komplexen Struktur wurden zwei verschiedene Präparationsverfahren für die $\mu$ ITO-Elektrode untersucht. Die $\mu$ ITO-Elektroden wurden entweder unter Standard-Luftbedingungen mit anschließender Plasma-Reinigung oder unter Argon-Atmosphäre gesintert. Diese Strategie wurde gewählt, da Sauerstoff-Fehlstellen im ITO die Leitfähigkeit und Elektronenmobilität verbessern sollen (Luo et al. 2006).

Abb. 4 zeigt einen Vergleich der nach den beiden Verfahren hergestellten Elektroden. Hier sind die Photostromdichten von $\mu$ ITO-PSI-Cyt$c$-Elektroden gegen die Anzahl der bei der Elektrodenherstellung verwendeten $\mu$ ITO-Schichten aufgetragen. Von 2 bis 8 Schichten steigt die Photostromdichte linear an. Das Verfahren unter Argon führt zu höheren Photoströmen $\left(17,5 \pm 0,4 \mu \mathrm{A} \mathrm{cm} \mathrm{cm}^{-2}\right.$ / Schicht, $\left.R^{2}=0,992, n=4\right)$. Das Sintern unter Luftatmosphäre ergibt ebenfalls definierte Photoströme, jedoch mit deutlich geringerem Stromertrag $\left(7,7 \pm 0,2 \mu \mathrm{A} \mathrm{cm}^{-2} /\right.$ Schicht, $\mathrm{R}^{2}=0,989$, $\mathrm{n}=4$ ). Die Proteinkonzentration (Cyt $c$ oder PSI) ist in beiden Strukturen sehr ähnlich und kann daher nicht der Grund für die drastische Veränderung der Photostromgröße sein. Die heterogene Elektronentransfergeschwindigkeitskonstante $\left(k_{s}\right)$ von Cyt $c$ an der $\mu$ ITO-Elektrode könnte möglicherweise der Grund für eine höhere Stromdichte der unter Argon hergestellten Elektroden des Photobiohybridsystems sein. Mit der Methode von Laviron (1979) wird ein $\mathrm{k}_{\mathrm{s}}$ von 1,1 $\pm 0,1 \mathrm{~s}^{-1}$ für Argon und 0,54 \pm $0,03 \mathrm{~s}^{-1}$ für Luft bestimmt. In diesem System muss folglich die Geschwindigkeit des Elektronentransfers der Elektrode auf Cyt $c$ ein limitierender Schritt in der Elektronentransportkette sein, der durch seine Verbesserung zu höheren Photoströmen führt.

Bei den nächsten Experimenten liegt der Fokus auf der Proteinmenge, die in die $\mu$ ITO-Struktur für verschiedene Schichten integriert werden kann. Die Konzentration von Cyt $c$ wurde mittels CV analysiert, während sich die PSI-Konzentration durch UV/VisAbsorptionsmessungen bzw. aus der Chlorophyllmenge, die aus der 
Elektrode extrahiert werden kann, bestimmen lässt. Abb. 5 zeigt die ermittelten Proteinkonzentrationen im Zusammenhang mit der geometrischen Fläche. Hierbei wurde eine lineare Beziehung sowohl zwischen der Cyt-c- als auch der PSI-Konzentration und der Anzahl der bei der Herstellung der Basiselektrode abgeschiedenen $\mu$ ITO-Schichten gefunden. Für PSI kann eine Konzentrationserhöhung bezogen auf die geometrische Fläche von 4,5 $\pm 0,2 \mathrm{pmol} \mathrm{cm}^{-2}$ / Schicht $\left(\mathrm{R}^{2}=0,997, \mathrm{n}=5\right)$ bestimmt werden, während der Wert für Cyt $c$ $0,46 \pm 0,01 \mathrm{nmol} \mathrm{cm}^{-2} /$ Schicht $\left(\mathrm{R}^{2}=\right.$ $0,998, \mathrm{n}=5$ ) erreicht.

In Bezug auf die elektroaktive Oberfläche (siehe Abb. 2) wird eine Oberflächenbedeckung von 0,2 $\pm 0,01$ pmol $\mathrm{cm}^{-2}$ für PSI berechnet, die nahe einer vollständigen Monolagen-Belegung liegt. Die gleiche Bestimmung für Cyt $c$ zeigt ebenfalls eine Monolagen-

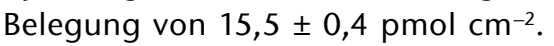
Diese Werte belegen, dass beide Proteine die gesamte 3D-Struktur durchdringen können, um so die volle elektroaktive Oberfläche der Elektrode zu nutzen. Die Elektronentransfereffizienz pro Molekül beschreibt die Umsatzzahl $\left(\mathrm{T}_{\mathrm{e}}\right)$. $\mathrm{T}_{\mathrm{e}}$ ist die Anzahl der Elektronen, die von jedem einzelnen Protein pro Sekunde übertragen werden. Das Ergebnis dieser Analyse ist in Abb. 5 dargestellt. Ab mindestens $4 \mathrm{x}-\mu$ ITO-Schichten wird $\mathrm{T}_{\mathrm{e}}$ für beide Proteine konstant, mit einem Wert von 0,42 $\pm 0,01 \mathrm{e}-$ Cyt $c^{-1}$ s-1 und $35 \pm$ $3 \mathrm{e}-\mathrm{PSI}^{-1} \mathrm{~s}^{-1}$.
Photophysikalische Eigenschaften von $\mu$ ITO-PSI-Cyt-c-Elektroden

In diesem Abschnitt charakterisieren wir die photophysikalischen Eigenschaften der $\mu$ ITO-PSI-Cyt-c-Elektrode, um ihr Potential als effiziente, biohybride und transparente Photokathode zu demonstrieren. In Abb. 6 ist die Photostromdichte einer $6 x-\mu$ ITO-PSI-Cyt$c$-Elektrode gegen die Lichtintensität aufgetragen. Die Michaelis-MentenGleichung kann für eine Auswertung der experimentellen Daten verwendet werden. Die einfallenden Photonen dienen als Substrat für das photoaktive PSI. Die Lichtintensität bei halbem Maximum $\left(K_{M}\right)$ ergibt 2,6 $\pm 0,2 \mathrm{~mW} \mathrm{~cm}^{-2}$, und ein maximaler Photostrom $\left(\mathrm{J}_{\max }\right)$ von $148 \pm 2 \mu \mathrm{A} \mathrm{cm}{ }^{-2}$ wird bestimmt. Die Kooperativität (n) der Lichtabhängigkeit beträgt 1,0 $\pm 0,1$. Dies bedeutet, dass die Anregung des PSI durch Photonen in Form paralleler, unabhängig auftretender Einzelereignisse stattfindet. Der aus diesen Experimenten abgeleitete $\mathrm{K}_{\mathrm{M}}$-Wert ist dem eines Cyt-c-/ PSI-Monoschichtsystems auf planaren Goldelektroden sehr ähnlich (Stieger et al. 2014) und um mehr als ein Faktor von 2 höher als bei einem Multischichtaufbau (Stieger et al. 2016).

Als nächstes Experiment wurde ein Photoactionspektrum gemessen und mit dem Absorptionsspektrum von PSI in Lösung und immobilisiert in der $\mu$ ITO-Struktur verglichen. Abb. 7 zeigt die auf den Peak bei $680 \mathrm{~nm}$ normierten Spektren. Das Photoactionspektrum folgt der gesamten Wellenlän- genverteilung von PSI in Lösung sowie für eine $6 x-\mu I T O-P S I-C y t-c$-Elektrode. Peaks werden bei 680,620 und 440 $\mathrm{nm}$ beobachtet, die jeweils der $\mathrm{Q}_{\mathrm{y}}$, $\mathrm{Q}_{\mathrm{x}-}$ und der Soret-Bande von Chlorophyll a zuzuordnen sind (Müh \& Zouni 2005). Aufgrund der Streuung und der Heterogenität von oberflächenassoziierten Proteinen ist die Absorption in der typischen Grünlücke des PSI der $\mu$ ITO-PSI-Cyt-c-Elektrode im Vergleich mit in Lösung befindlichen Proteinen höher. Bei kürzeren Wellenlängen verringert sich der Photostrom analog zum Absorptionsverhalten. Aufgrund der anderen immobilisierten Komponente Cyt $c$ und der hohen Proteinbeladung senken die Soret-Absorptionen bei $410 \mathrm{~nm}$ (ferri) und $416 \mathrm{~nm}$ (ferro) die effektive Photonen-zu-Ladungsträger-Umwandlung von PSI. Aus den Transmissionsspektren von ITO und $\mu$ ITO ist eine Abnahme der Transmission über das sichtbare Lichtspektrum bei kürzeren Wellenlängen erkennbar. Werden beide Spektren verglichen, reduziert sich die Transmission bei $680 \mathrm{~nm}$ von $86 \%$ auf $11 \%$ für die 3DElektrode (Daten nicht gezeigt). Dies deutet auf einen erhöhten Streueffekt im $\mu$ ITO hin.

Die Quanteneffizienz wurde für verschiedene Lichtintensitäten der $\mu \mathrm{ITO}$ PSI-Cyt-c-Elektrode berechnet. Hierbei wird die einfallende Licht-zu-StromEffizienz als externe Quanteneffizienz (EQE) bezeichnet und ein Spitzenwert von etwa $11 \%$ (6,6 \% am apparenten $K_{M}$ ) bestimmt. Die interne Quanteneffizienz (IQE) repräsentiert das
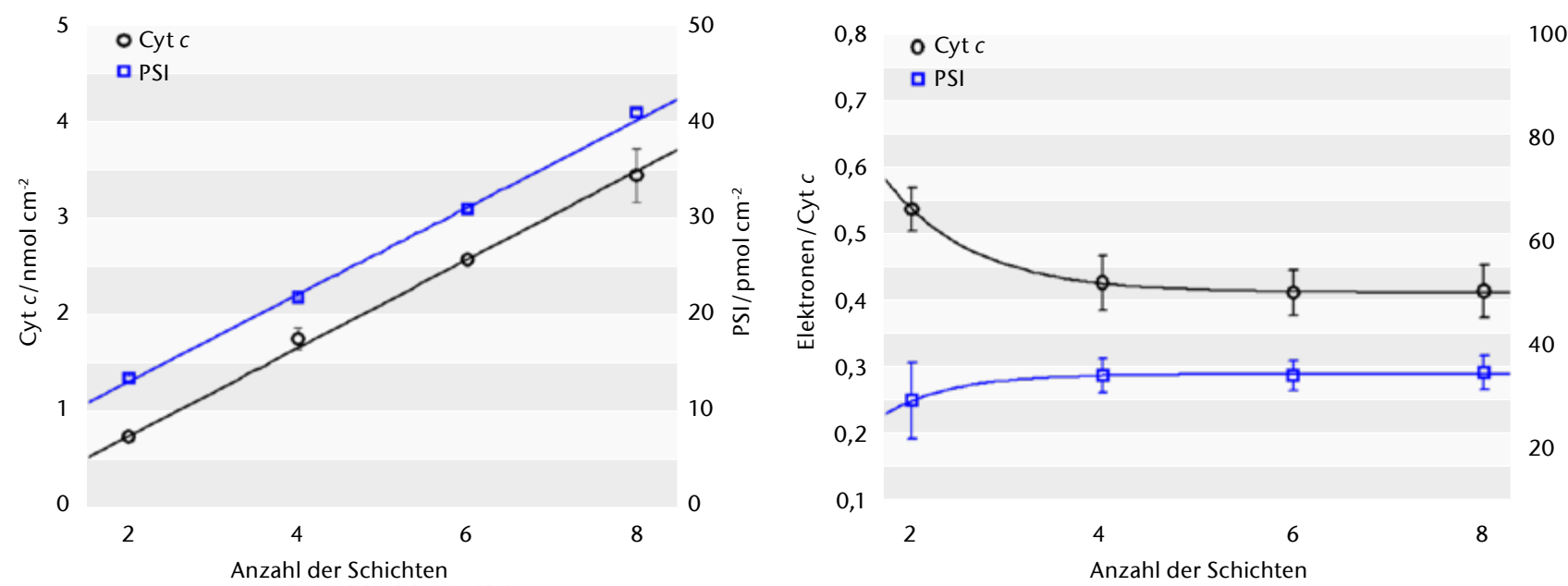

Abb. 5) Proteinkonzentration (links) und Umsatzzahl (rechts) in Abhängigkeit von der Anzahl der $\mu$ ITO-Schichten. Es kann eine lineare Beziehung zwischen der Proteinmenge und der Anzahl der Schichten gefunden werden (PSI: 4,5 $\pm 0,2 \mathrm{pmol} \mathrm{cm}^{-2} /$ Schicht, $R^{2}=0,997 ; C y t c: 0,46 \pm 0,01 \mathrm{nmol} \mathrm{cm}^{-2} / \mathrm{Schicht}, R^{2}=0,998 ; n=4$ ). Geometrische Elektrodenfläche: $0,2 \mathrm{~cm}^{2}$. 
Verhältnis zwischen der Anzahl der absorbierten Photonen zu der Anzahl der erzeugten Ladungsträger in einer gegebenen Zeit. Die Anzahl absorbierter Photonen kann ferner aus dem Absorptionsspektrum einer $\mu$ ITO-PSICyt-c-Elektrode und dem Lichtquellenspektrum berechnet werden. Die Berechnung berücksichtigt nicht, dass aufgrund der Lichtstreuung der mesoporösen Struktur die reale Absorption der Elektrode geringer ist. Eine Auftragung der IQE gegen die Lichtleistung zeigt einen Spitzenwert bei niedrigen Lichtintensitäten von etwa $30 \%$ (und $18 \%$ am apparenten $\mathrm{K}_{\mathrm{M}}$ ). Diese Werte stellen momentan eine der höchsten gemessenen Quanteneffizienzen von PSI-Elektroden dar (Yehezkeli et al. 2010; Badura et al. 2011a; Gordiichuk et al. 2014; Beam et al. 2015; Gizzie et al. 2015; Stieger et al. 2016).

\section{Schlussfolgerungen}

In dieser Studie wurde eine skalierbare mesoporöse 3D-Elektrodenstruktur erfolgreich mit PSI kombiniert, welches mithilfe des Redoxproteins Cyt $c$ elektrisch kontaktiert wird und zu einer hocheffizienten Photokathode führt. Die Experimente deuten auf eine lineare Skalierbarkeit des Photostroms über verschiedene Schichtdicken der $\mu$ ITOElektrode bis zu $40 \mu \mathrm{m}$ hin. Die Dicke des $\mu$ ITO kann bei der Präparation der

$-160$

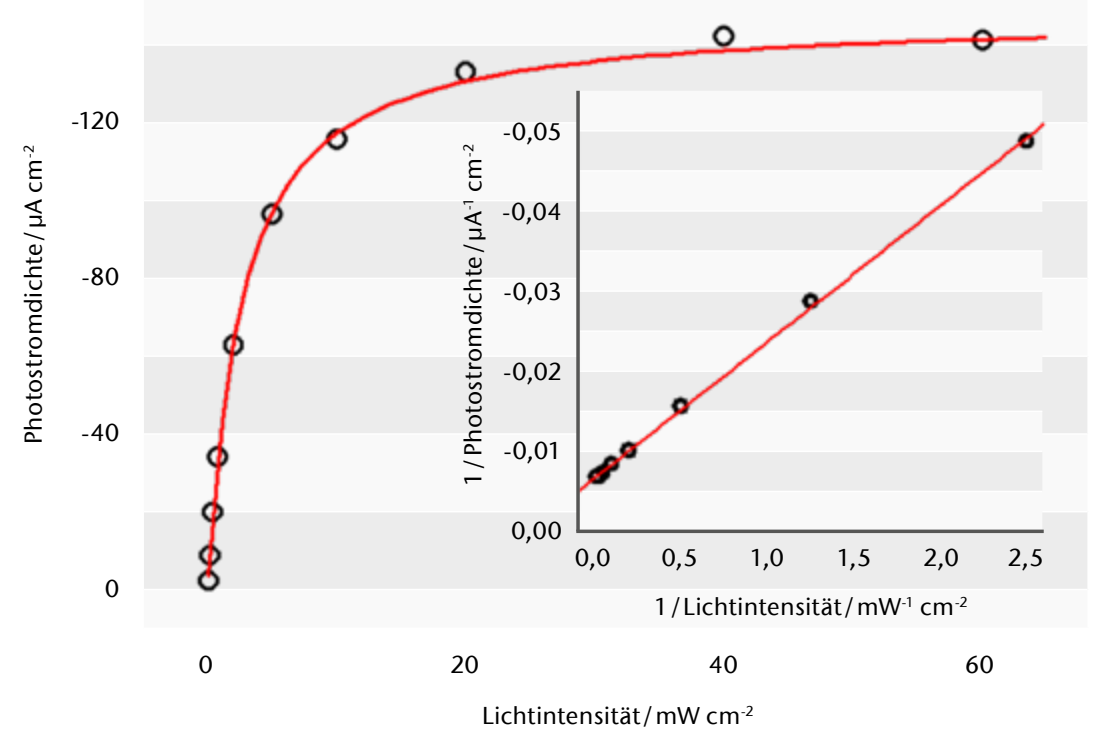

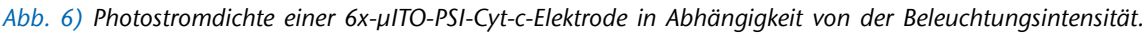
Die Daten wurden durch Regression mit der Michaelis-Menten-Gleichung $\left(K_{\mathrm{M}}=2,6 \pm 0,2 \mathrm{~mW} \mathrm{\textrm {cm } ^ { - 2 }}, J_{\max }=148 \pm\right.$ $\left.2 \mu \mathrm{Acm}^{-2}, n=1,0 \pm 0,1, R^{2}=0,998\right)$ angepasst. Einsatz: Lineweaver-Burk Plot der gleichen Daten. Experimente wurden bei Raumtemperatur in Phosphatpuffer ( $5 \mathrm{mM}, \mathrm{pH}$ 7) unter Verwendung von weißem Licht bei einem Potential von -100 mV vs. $\mathrm{Ag} \mid \mathrm{AgCl}(n=3)$ durchgeführt. Geometrische Elektrodenfläche: $0,2 \mathrm{~cm}^{2}$.

Elektrode durch die Anzahl der SpinCoating-Schritte leicht und reproduzierbar kontrolliert werden, während die elektroaktive Oberfläche der Dicke der 3D-Struktur linear folgt. Durch die schnelle und einfache Präparation von $\mu$ ITO lässt sich die Produktionsgeschwindigkeit solcher Elektroden im Vergleich zu anderen Präparationsprotokollen deutlich erhöhen. Darüber hi- naus ermöglicht das Studium verschiedener Fertigungsverfahren ein tieferes Verständnis der Funktionalität und der limitierenden Elektronentransferschritte der Photobiohybridstruktur. Ferner können PSI und Cyt $c$ gut in die 3D-Elektrodenstruktur integriert werden. Bemerkenswert ist, dass die gesamte innere Elektrodenoberfläche zur Proteinimmobilisierung und Pho-
1,5

Absorptionsspektrum PSI

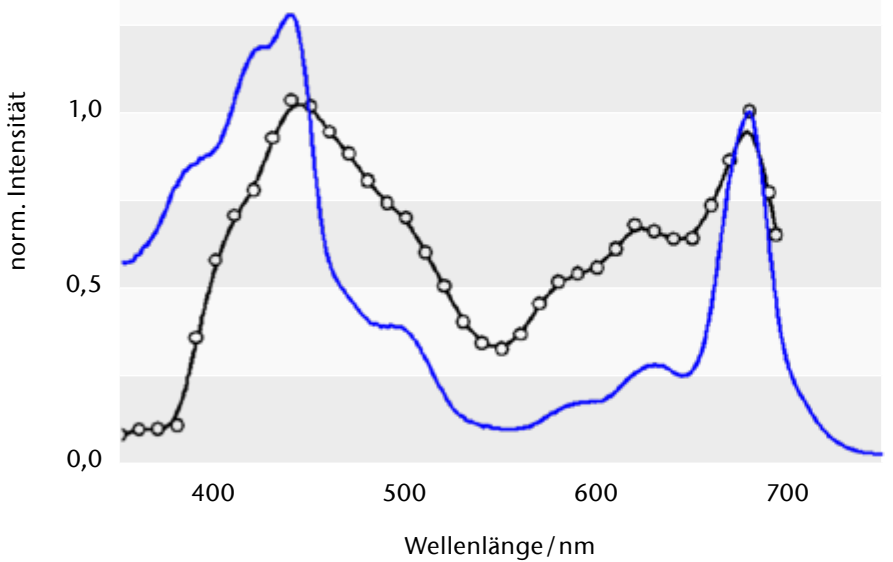

40

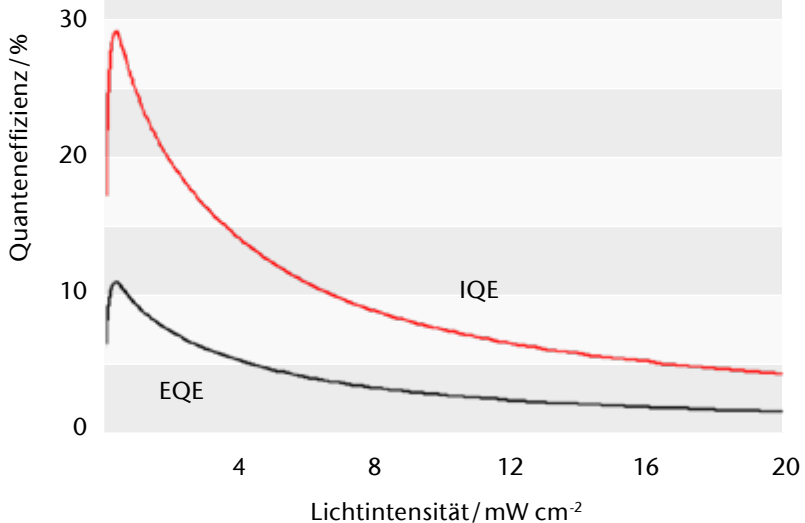

Abb. 7) (Links) Photoactionspektrum einer 6x- $\mu$ ITO-PSI-Cyt-c-Elektrode im Vergleich zum Absorptionsspektrum von PSI in Phosphatpuffer (5 mM, pH 7), normalisiert auf den Peak bei $680 \mathrm{~nm}$. Das Photoactionspektrum wurde mit monochromatischem Licht (Bandbreite $15 \mathrm{~nm}$, Intensitäten $<10 \mathrm{~mW} \mathrm{~cm}^{-2}$ ) in aerobem Phosphatpuffer (5 mM, $\mathrm{pH}$ 7) erhalten. Die Spektren sind die Mittelwerte von drei unabhängigen Messungen. (Rechts) Externe (EQE) und interne (IQE) Quanteneffizienzen einer 6x- $\mu$ ITO-PSI-Cytc-Elektrode, berechnet für unterschiedliche Lichtintensitäten. 
tostrombildung genutzt werden kann. Auf dieser Basis ist es möglich, hohe kathodische Photoströme zu erzeugen, die mit der Anzahl von $\mu$ ITO-Schichten skalierbar sind. Das System zeigt hohe Quanteneffizienzen und folgt den spektralen Eigenschaften von PSI. Der Biohybrid-Ansatz ermöglicht den Aufbau von komplexeren Licht zu Strom umwandelnden Elektroden, z. B. durch die Integration von weiteren Enzymen oder Nanomaterialien für die Umwandlung von Licht in chemische Energie. Die Studie zeigt außerdem, dass das Cyt-c-PSI-System auf eine Vielzahl von Materialien und Oberflächen aufgebracht werden kann und Photostromdichten von mehr als 150 $\mu \mathrm{A} \mathrm{cm}{ }^{-2}$ möglich sind.

\section{Danksagungen}

Wir bedanken uns für die Unterstützung dieser Forschung durch das Bundesministerium für Bildung und Forschung (BMBF), Deutschland (Biotechnologie 2020+, Projekte: 031A154A+B).

\section{LITERATUR}

Araci ZO, Runge AF, Doherty WJ, Saavedra SS (2008) Correlating molecular orientation distributions and electrochemical kinetics in subpopulations of an immobilized protein film. J Am Chem Soc 130:1572-1573. doi: 10.1021/ja710156d

Badura A, Guschin D, Kothe T (2011a) Photocurrent generation by photosystem 1 integrated in crosslinked redox hydrogels. Energy Environ Sci 4:2435. doi: 10.1039/c1ee01126j

Badura A, Kothe T, Schuhmann W, Rögner M (2011b) Wiring photosynthetic enzymes to electrodes. Energy Environ Sci 4:3263. doi: 10.1039/c1ee01285a

Baker DR, Simmerman RF, Sumner JJ (2014) Photoelectrochemistry of photosystem I bound in nafion. Langmuir 30:13650-5. doi: 10.1021/la503132h

Beam JC, LeBlanc G, Gizzie EA (2015) Construction of a Semiconductor-Biological Interface for Solar Energy Conversion: P-Doped Silicon/Photosystem I/Zinc Oxide. Langmuir 31:10002-10007. doi: 10.1021/acs. langmuir.5b02334

Carmeli I, Frolov L, Carmeli C, Richter S (2007) Photovoltaic Activity of Photosystem I-Based Self-Assembled Monolayer. J Am Chem Soc 129:12352-12353. doi: $10.1021 / \mathrm{ja} 073040 \mathrm{c}$

Ciesielski PN, Faulkner CJ, Irwin MT (2010) Enhanced Photocurrent Production by Photosystem I Multilayer Assemblies. Adv Funct Mater 20:4048-4054. doi: 10.1002/adfm.201001193

Ciesielski PN, Scott AM, Faulkner CJ (2008) Functionalized nanoporous gold leaf electrode films for the immobilization of photosystem I. ACS Nano 2:2465-72. doi: $10.1021 / \mathrm{nn} 800389 \mathrm{k}$
Ciobanu M, Kincaid HA, Lo V (2007) Electrochemistry and photoelectrochemistry of photosystem I adsorbed on hydroxyl-terminated monolayers. J Electroanal Chem 599:72-78. doi: 10.1016/j.jelechem.2006.09.019

Cramer WA, Zhang H, Yan J (2006) Transmembrane traffic in the cytochrome b6f complex. Annu Rev Biochem 75:769-90. doi: 10.1146/annurev.biochem.75.103004.142756

Czechowski N, Lokstein H, Kowalska D (2014) Large plasmonic fluorescence enhancement of cyanobacterial photosystem I coupled to silver island films. Appl Phys Lett 105:43701. doi: 10.1063/1.4891856

Díaz-Quintana A, Leibl W, Bottin H, Sétif P (1998) Electron transfer in photosystem I reaction centers follows a linear pathway in which iron-sulfur cluster $F B$ is the immediate electron donor to soluble ferredoxin. Biochemistry 37:3429-39. doi: 10.1021/bi972469|

Dominguez-Benetton X, Srikanth S, Satyawali Y (2013) Enzymatic Electrosynthesis: An Overview on the Progress in Enzyme- Electrodes for the Production of Electricity, Fuels and Chemicals. J Microb Biochem Technol S6:7. doi: 10.4172/1948-5948.S6-007

Efrati A, Tel-Vered R, Michaeli D (2013) Cytochrome c-coupled photosystem I and photosystem II (PSI/PSII) photo-bioelectrochemical cells. Energy Environ Sci 6:2950. doi: $10.1039 /$ c3ee41568f

El Kasmi A, Leopold MC, Galligan R (2002) Adsorptive immobilization of cytochrome $c$ on indium/tin oxide (ITO): Electrochemical evidence for electron transfer-induced conformational changes. Electrochem commun 4:177-181. doi: 10.1016/S1388-2481(01)00299-5

Feifel SC, Stieger KR, Lokstein H (2015) High photocurrent generation by photosystem I on artificial interfaces composed of $\pi$-system-modified graphene. J Mater Chem A 3:12188-12196. doi: 10.1039/C5TA00656B

Frasca S, Molero Milan A, Guiet A (2013) Bioelectrocatalysis at mesoporous antimony doped tin oxide electrodes - Electrochemical characterization and direct enzyme communication. Electrochim Acta 110:172-180. doi: 10.1016/j.electacta.2013.03.144

Frasca S, von Graberg T, Feng IJ (2010) Mesoporous Indium Tin Oxide as a Novel Platform for Bioelectronics. ChemCatChem 2:839-845. doi: 10.1002/ cctc. 201000047

Friebe VM, Delgado JD, Swainsbury DJK (2016) Plasmon-Enhanced Photocurrent of Photosynthetic Pig ment Proteins on Nanoporous Silver. Adv Funct Mater 26:285-292. doi: 10.1002/adfm.201504020

Gizzie EA, LeBlanc G, Jennings GK, Cliffel DE (2015) Electrochemical Preparation of Photosystem I-Polyaniline Composite Films for Biohybrid Solar Energy Conversion. ACS Appl Mater Interfaces 7:9328-9335. doi: 10.1021/acsami.5b01065

Gordiichuk PI, Wetzelaer G-JAH, Rimmerman D (2014) Solid-State Biophotovoltaic Cells Containing Photosystem I. Adv Mater 26:4863-4869. doi: 10.1002 adma.201401135

Grimme RA, Lubner CE, Bryant DA, Golbeck JH (2008) Photosystem I/molecular wire/metal nanoparticle bioconjugates for the photocatalytic production of $\mathrm{H} 2$. J Am Chem Soc 130:6308-9. doi: 10.1021/ja800923y

Grimme RA, Lubner CE, Golbeck JH (2009) Maximizing $\mathrm{H} 2$ production in Photosystem I/dithiol molecular wire/ platinum nanoparticle bioconjugates. Dalton Trans 10106-13. doi: 10.1039/b909137h

Grotjohann I, Fromme P (2005) Structure of cyanobac terial photosystem I. Photosynth Res 85:51-72. doi: 10.1007/s11120-005-1440-4

Hartmann V, Kothe T, Pöller S (2014) Redox hydrogels with adjusted redox potential for improved efficiency in Z-scheme inspired biophotovoltaic cells. Phys Chem Chem Phys 16:11936-41. doi: 10.1039/c4cp00380b
Jordan P, Fromme P, Witt HT (2001) Three-dimensional structure of cyanobacterial photosystem I at $2.5 \mathrm{~A}$ resolution. Nature 411:909-17. doi: 10.1038/35082000

Kato M, Cardona T, Rutherford AW, Reisner E (2012) Photoelectrochemical water oxidation with photosystem II integrated in a mesoporous indium-tin oxide electrode. J Am Chem Soc 134:8332-5. doi: 10.1021/ ja301488d

Kincaid HA, Niedringhaus T, Ciobanu M (2006) Entrapment of photosystem I within self-assembled films. Langmuir 22:8114-20. doi: 10.1021/la061326+

Ko BS, Babcock B, Jennings GK (2004) Effect of Surface Composition on the Adsorption of Photosystem I onto Alkanethiolate Self-Assembled Monolayers on Gold. Langmuir 20:4033-38. doi: 10.1021/la0356809

Kothe T, Plumeré N, Badura A (2013) Combination of a photosystem 1-based photocathode and a photosystem 2-based photoanode to a Z-scheme mimic for biophotovoltaic applications. Angew Chem Int Ed Engl 52:14233-6. doi: 10.1002/anie.201303671

Kothe T, Pöller S, Zhao F (2014) Engineered electrontransfer chain in photosystem 1 based photocathodes outperforms electron-transfer rates in natural photosynthesis. Chemistry 20:11029-34. doi: 10.1002/ chem.201402585

Laviron E (1979) General expression of the linear potential sweep voltammogram in the case of diffusionless electrochemical systems. IElectroanal Chem Interfacial Electrochem 101:19-28. doi: 10.1016/S00220728(79)80075-3

LeBlanc G, Chen G, Gizzie E (2012) Enhanced photocurrents of photosystem I films on p-doped silicon. Adv Mater 24:5959-62. doi: 10.1002/adma.201202794

LeBlanc G, Gizzie E, Yang S (2014) Photosystem I Protein Films at Electrode Surfaces for Solar Energy Conversion. Langmuir 30:10990-11001. doi: 10.1021/la500129q

Leech D, Kavanagh P, Schuhmann W (2012) Enzymati fuel cells: Recent progress. Electrochim Acta 84:223234. doi: 10.1016/j.electacta.2012.02.087 Liu Y, Peters K, Mandlmeier B (2014) Electrochimica Acta Macroporous indium tin oxide electrode layers as conducting substrates for immobilization of bulky electroactive guests. Electrochim Acta 140:108-115. doi: 10.1016/j.electacta.2014.05.046

Luo SN, Kono A, Nouchi N, Shoji F (2006) Effective creation of oxygen vacancies as an electron carrier source in tin-doped indium oxide films by plasma sputtering. I Appl Phys 100:113701. doi: 10.1063/1.2372571

Manocchi AK, Baker DR, Pendley SS (2013) Photocurrent generation from surface assembled photosystem I on alkanethiol modified electrodes. Langmuir 29:2412-9. doi: 10.1021/la304477u

Mersch D, Lee CY, Zhang JZ (2015) Wiring of Photosys tem II to Hydrogenase for Photoelectrochemical Water Splitting. J Am Chem Soc 137:8541-9. doi: 10.1021/ jacs.5b03737

Mershin A, Matsumoto K, Kaiser L (2012) Self-assembled photosystem-I biophotovoltaics on nanostructured TiO(2 )and ZnO. Sci Rep 2:234. doi: 10.1038/srep00234

Müh F, Zouni A (2005) Extinction coefficients and critical solubilisation concentrations of photosystems I and II from Thermosynechococcus elongatus. Biochim Biophys Acta 1708:219-28. doi: 10.1016/j. bbabio. 2005.03.005

Nelson N, Yocum CF (2006) Structure and function of photosystems I and II. Annu Rev Plant Biol 57:521-65. doi: 10.1146/annurev.arplant.57.032905.105350

Nguyen K, Bruce BD (2014) Growing green electricity: progress and strategies for use of photosystem I for sustainable photovoltaic energy conversion. Biochim Biophys Acta 1837:1553-66. doi: 10.1016/j.bbabio.2013.12.013 
Nicholson RS, Shain I (1964) Theory of Stationary Electrode Polarography. Single Scan and Cyclic Methods Applied to Reversible, Irreversible, and Kinetic Systems. Anal Chem 36:706-723. doi: 10.1021/ac60210a007

Ocakoglu K, Krupnik T, van den Bosch B (2014) Photosystem I-based Biophotovoltaics on Nanostructured Hematite. Adv Funct Mater 24:7467-7477. doi: 10.1002/ adfm.201401399

Operamolla A, Ragni R, Milano F (2015) “Garnishing" the photosynthetic bacterial reaction center for bioelec tronics. J Mater Chem C 3:6471-6478. doi: 10.1039/ C5TC00775E

Plumeré N (2012) Single molecules: A protein in the spotlight. Nat Nanotechnol 7:616-7. doi: 10.1038/ nnano.2012.175

Sarauli D, Peters K, Xu C (2014a) 3D-electrode architectures for enhanced direct bioelectrocatalysis of pyrroloquinoline quinone-dependent glucose dehydrogenase. ACS Appl Mater Interfaces 6:17887-93. doi: 10.1021/ am5046026

Sarauli D, Xu C, Dietzel B (2014b) A multilayered sulfonated polyaniline network with entrapped pyrrolo quinoline quinone-dependent glucose dehydrogenase: tunable direct bioelectrocatalysis. I Mater Chem B 2:3196-3203. doi: 10.1039/c4tb00336e

Sassolas A, Blum LJ, Leca-Bouvier BD (2012) Immobilization strategies to develop enzymatic biosensors. Biotechnol Adv 30:489-511. doi: 10.1016/j.biotechadv.2011.09.003

Schaming D, Renault C, Tucker RT (2012) Spectroelec trochemical characterization of small hemoprotein adsorbed within nanostructured mesoporous ITO electrodes. Langmuir 28:14065-72. doi: 10.1021/la302913j

Shah VB, Henson WR, Chadha TS (2015) Linker-free deposition and adhesion of Photosystem I onto nanos- tructured $\mathrm{TiO} 2$ for biohybrid photoelectrochemical cells. Langmuir 31:1675-82 doi: 10.1021/la503776b Stieger KR, Ciornii D, Kölsch A (2016) Engineering of supramolecular photoactive protein architectures: the defined co-assembly of photosystem I and cytochrome c using a nanoscaled DNA-matrix. Nanoscale 8:1069510705. doi: 10.1039/C6NR00097E

Stieger KR, Feifel SC, Lokstein H, Lisdat F (2014) Advanced unidirectional photocurrent generation via cytochrome $c$ as reaction partner for directed assembly of photosystem I. Phys Chem Chem Phys 16:15667-74. doi: $10.1039 / \mathrm{c} 4 \mathrm{cp} 00935$

Terasaki N, Yamamoto N, Hiraga T (2009) Plugging a molecular wire into photosystem I: reconstitution of the photoelectric conversion system on a gold electrode. Angew Chem Int Ed Engl 48:1585-7. doi: 10.1002/ anie. 200805748

Terasaki N, Yamamoto N, Hiraga T (2006) Fabrication of novel photosystem I-gold nanoparticle hybrids and their photocurrent enhancement. Thin Solid Films 499:153-156. doi: 10.1016/j.tsf.2005.07.050

Wang F, Liu X, Willner I (2013) Integration of photoswitchable proteins, photosynthetic reaction centers and semiconductor/biomolecule hybrids with electrode supports for optobioelectronic applications. Adv Mater 25:349-77. doi: 10.1002/adma.201201772

Yan X, Faulkner CJ, Jennings GK, Cliffel DE (2012) Photosystem I in Langmuir-Blodgett and Langmuir-Schaefer monolayers. Langmuir 28:15080-6. doi: 10.1021/ la302611a

Yehezkeli O, Tel-Vered R, Michaeli D (2013) Photosystem I (PSI)/Photosystem II (PSII)-based photo-bioelectrochemical cells revealing directional generation of photocurrents. Small 9:1-9. doi: 10.1002/smll.201300051
Yehezkeli O, Wilner OI, Tel-Vered R (2010) Generation of photocurrents by bis-aniline-cross-linked Pt nanoparticle/photosystem I composites on electrodes. J Phys Chem B 114:14383-8. doi: 10.1021/jp100454u

Yu D, Wang M, Zhu G (2015) Enhanced photocurrent production by bio-dyes of photosynthetic macromolecules on designed TiO2 film. Sci Rep 5:9375. doi: 10.1038/srep09375

Zhao F, Conzuelo F, Hartmann V (2015) Light Induced $\mathrm{H} 2$ Evolution from a Biophotocathode Based on Photosystem 1 - Pt Nanoparticles Complexes Integrated in Solvated Redox Polymers Films. J Phys Chem B 119:13726-31. doi: 10.1021/acs.jpcb.5b03511

\section{AUTOREN}

Prof. Dr. habil. Fred Lisdat Dipl. NanoSc. Kai Stieger Dr. Sven Christian Feifel Biosystemtechnik Technische Hochschule Wildau

Dr. Heiko Lokstein

Department of Chemical Physics and Optics Charles University Prague, Czech Republic

Prof. Dr. Athina Zouni

Dr. Mahdi Hejazi

Institut für Biologie

Humboldt-Universität zu Berlin

E-Mail für Korrespondenz:

fred.lisdat@th-wildau.de

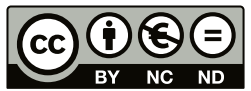

